\title{
Euler Equation for Descriptors of the Spherically Symmetric Coulomb Systems
}

\author{
Á. Nagy \\ Department of Theoretical Physics, University of Debrecen, H-4010 Debrecen, Hungary
} (Dated: November 26, 2015)

\begin{abstract}
Euler equations for a family of descriptors of the spherically symmetric Coulomb systems are derived and discussed. Generalized Weizsäcker and Pauli energies and potentials are introduced.
\end{abstract}




\section{INTRODUCTION}

According to the density functional theory, the ground-state electron density is a fundamental quantity, as it determines every property of the electron system [1]. In case of a Coulomb system, not only the electron density possesses this fundamental property. Namely, it turned out that other quantities are also capable of fully determining every property of a Coulomb system. The first one that proved to be a descriptor of a Coulomb system was the shape function (density per particle )[2]. Later, reactivity indicators [3] such as Fukui function, local softness, softness kernel, electrostatic potential, local kinetic energy and local temperature $[4,5]$ were also shown to be adequate for describing a Coulomb system. Then a whole family of descriptors for Coulomb systems have been presented [6, 7]. Recently, it has been proved that the local Shannon information is also capable of fully determining every property of a Coulomb system [8].

Though any of these descriptors is able to determine a Coulomb system, they are not so fundamental in calculations as the density. They are generally obtained from standard Kohn-Sham calculations. Equations capable computing these descriptors directly, have not been presented. In this paper, such equations are derived for spherically symmetric systems. These correspond to the Euler equation of the standard density functional theory.

Density functional calculations are frequently done in the Kohn-Sham scheme, though in principle orbital-free calculations [9-11] would be also possible. Because of the lack of accurate approximation for the kinetic energy functional, these calculations are not accurate enough. Recently an approach has been proposed to solve the orbital-free problem for spherically symmetric systems [12-15]. In this paper, that approach is generalized to derive Euler equations for a family of descriptors of Coulomb systems.

In the following section the definition and main properties of these descriptors of Coulomb systems are summarized. In Section III differential equation is derived for spherically symmetric systems. Section IV presents Euler equations for the descriptors. The last section is devoted to discussion. 


\section{II. "GENERALIZED DENSITIES" OF COULOMB SYSTEMS}

The descriptors of Coulomb systems that we call "generalized densities" are defined with the Kohn-Sham one-partical densities. The Kohn-Sham equations [17] can be written as

$$
-\frac{1}{2} \nabla^{2} u_{k}(\mathbf{r})+v_{\mathrm{KS}}(\mathbf{r}) u_{k}(\mathbf{r})=\epsilon_{k} u_{k}(\mathbf{r})
$$

where $v_{\mathrm{KS}}(\mathbf{r}), u_{k}(\mathbf{r})$ and $\epsilon_{k}$ are the Kohn-Sham potential, the orbitals and the orbital energies, respectively. The "generalized density" $\xi(\mathbf{r})$ is defined by the expression $[6,7]$

$$
\xi(\mathbf{r})=\sum_{k}^{N} \alpha_{k} n_{k}(\mathbf{r}),
$$

where $\alpha_{k} \geq 0$ for $1 \leq k<N$ and $\alpha_{N}>0$.

$$
n_{k}(\mathbf{r})=\left|u_{k}(\mathbf{r})\right|^{2}
$$

are the one-partical densities. The sum is going for the occupied orbitals and $N$ is the number of electrons. The notation "generalized densities" refers to the fact that this family of descriptors contains the density as a special case: if $\alpha_{k}$ are the occupation numbers, that is, $\alpha_{k}=\lambda_{k}$, then $\xi$ is the electron density. The shape function $\sigma$ also belongs to this family: if $\alpha_{k}=\lambda_{k} / N, \xi(\mathbf{r})$ is the shape function: $\xi(\mathbf{r})=\sigma(\mathbf{r})=n(\mathbf{r}) / N$. Another interesting case is the highest occupied orbital density: if $\alpha_{1}=\ldots=\alpha_{N-1}=0$ and $\alpha_{N}=1$, $\xi(\mathbf{r})=n_{\text {HOMO }}(\mathbf{r}) \approx f^{-}(\mathbf{r})$ which approximates the Fukui function. It is worth mentioning [16] that the frontier molecular orbitals (highest occupied and lowest unoccupied orbitals) determine the electron density, and thus all properties of a system (even if the system is not Coulombic).

It was shown in [6] that the generalized densities (2) are descriptors of a Coulomb system, that is, capable of determining all its properties. The following theorem was proved: For any Coulomb system, $\xi(\mathbf{r})$ determines the external potential $v(\mathbf{r})$ up to an additive constant. $\xi(\mathbf{r})$ also determines the number of electron. The proof based on the cusp condition and the asymptotic behavour of the generalized density, can be found in Ref. [6]. 


\section{DIFFERENTIAL EQUATION FOR SPHERICALLY SYMMETRIC GENER- ALIZED DENSITIES}

In spherically symmetric Coulomb systems, it is convenient to introduce the radial generalized density $\tilde{\xi}(r)=4 \pi r^{2} \xi(r)$. Though the derivation is done in the Kohn-Sham scheme, the results will have an orbital-free form. The Kohn-Sham equations (1) for spherically symmetric systems take the form $[6]$

$$
-\frac{1}{2} \frac{d^{2} P_{i}}{d r^{2}}+\frac{l_{i}\left(l_{i}+1\right)}{2 r^{2}} P_{i}+v_{K S} P_{i}=\epsilon_{i} P_{i}
$$

where $P_{i}(r)=r R_{i}(r)$ and $l_{i}$ are the radial wave functions and the azimuthal quantum numbers, respectively. Then the radial generalized density takes the form

$$
\tilde{\xi}=\sum_{i} \alpha_{i} \varrho_{i}
$$

where $\varrho_{i}(r)=4 \pi r^{2} n_{i}(r)$ is the one-particle radial density. Eq. (5) can be rewritten as

$$
\tilde{\xi}=\sum_{i} a_{i} w_{i} \varrho_{i}
$$

where

$$
a_{i}=\frac{\alpha_{i}}{w_{i}}
$$

In the following the weighting factors $w_{i}$ are selected as

$$
w_{i}=e^{\beta \varepsilon_{i}-\gamma l_{i}\left(l_{i}+1\right)}
$$

with any real values $\beta$ and $\gamma$.

Dividing Eq. (4) by $P_{i}$, differentiating, then multiplying by $P_{i}^{2}$ we obtain

$$
-\frac{1}{2} P_{i} P_{i}^{\prime \prime \prime}+\frac{1}{2} P_{i}^{\prime} P_{i}^{\prime \prime}-\frac{l_{i}\left(l_{i}+1\right)}{r^{3}} P_{i}^{2}+v_{K S}^{\prime} P_{i}^{2}=0 .
$$

' denotes the derivation with respect to the radial coordinate $r$. Multiplying Eq. (4) by $P_{i}^{\prime}$ and combining it with Eq. (9) we arrive at

$$
P_{i} P_{i}^{\prime \prime \prime}+3 P_{i}^{\prime} P_{i}^{\prime \prime}=2 \frac{l_{i}\left(l_{i}+1\right)}{r^{2}} \varrho_{i}^{\prime}++4 v_{K S}^{\prime} \varrho_{i}^{\prime}-4 \epsilon_{i} \varrho_{i}^{\prime}-\frac{l_{i}\left(l_{i}+1\right)}{r^{3}} \varrho_{i}+2 v_{K S}^{\prime} \varrho_{i}=0
$$

where $\varrho_{i}=P_{i}^{2}$. 
The third derivative of $\tilde{\xi}(r)$ is

$$
\tilde{\xi}^{\prime \prime \prime}=2 \sum_{i} w_{i} a_{i}\left[P_{i} P_{i}^{\prime \prime \prime}+3 P_{i}^{\prime} P_{i}^{\prime \prime}\right]
$$

Taking into account that

$$
\begin{gathered}
\tilde{\xi}^{\prime}=\sum_{i} w_{i} a_{i} \varrho_{i}^{\prime}, \\
\frac{\partial \tilde{\xi}^{\prime}}{\partial \beta}=\sum_{i} w_{i} a_{i} \epsilon_{i} \varrho_{i}^{\prime}
\end{gathered}
$$

and

$$
\frac{\partial \tilde{\xi}}{\partial \gamma}=-\sum_{i} w_{i} a_{i} l_{i}\left(l_{i}+1\right) \varrho_{i}
$$

the third derivative of $\tilde{\xi}(r)$ can be written as

$$
\tilde{\xi}^{\prime \prime \prime}=8 v_{K S} \tilde{\xi}^{\prime}+4 v_{K S}^{\prime} \tilde{\xi}-8 \frac{\partial \tilde{\xi}^{\prime}}{\partial \beta}+\frac{4}{r^{2}} \frac{\partial \tilde{\xi}^{\prime}}{\partial \gamma}-\frac{4}{r^{3}} \frac{\partial \tilde{\xi}}{\partial \gamma} .
$$

Note that Eq. (15) is valid for any $\tilde{\xi}(r)$, that is, for any values of $a_{i}$.

\section{EULER EQUATION FOR THE GENERALIZED DENSITIES}

Define a generalized Weizsäcker expression

$$
S_{w}=\frac{1}{8} \int \frac{(\nabla \xi(\mathbf{r}))^{2}}{\xi(\mathbf{r})} d \mathbf{r}
$$

The functional derivative of $S_{w}$ with respect to $\xi(\mathbf{r})$ is given by

$$
\frac{\delta S_{w}}{\delta \xi}=\frac{1}{8}\left(\frac{\nabla \xi}{\xi}\right)^{2}-\frac{1}{4} \frac{\nabla^{2} \xi}{\xi} .
$$

It can also be written as

$$
\frac{\delta S_{w}}{\delta \xi}=\frac{1}{8}\left(\frac{\tilde{\xi}^{\prime}}{\tilde{\xi}}\right)^{2}-\frac{1}{4} \frac{\tilde{\xi}^{\prime \prime}}{\tilde{\xi}}
$$

or

$$
\frac{\delta S_{w}}{\delta \xi}=-\frac{1}{2} \frac{\nabla^{2} \xi^{1 / 2}}{\xi^{1 / 2}}
$$


Define a generalized potential as

$$
u=\frac{1}{2} \frac{\nabla^{2} \xi^{1 / 2}}{\xi^{1 / 2}}-v_{K S}
$$

The Kohn-Sham potential can be given as

$$
v_{K S}=-\frac{1}{8}\left(\frac{\tilde{\xi}^{\prime}}{\tilde{\xi}}\right)^{2}+\frac{1}{4} \frac{\tilde{\xi}^{\prime \prime}}{\tilde{\xi}}-u .
$$

Differentiating $v_{K S}(r)$ with respect to $r$, then substituting $v_{K S}(r)$ and $v_{K S}^{\prime}(r)$ into equation Eq. (15) we arrive at

$$
\frac{1}{2} \tilde{\xi} u^{\prime}+\tilde{\xi}^{\prime} u=F
$$

where

$$
F=-\frac{\partial \tilde{\xi}^{\prime}}{\partial \beta}-\frac{1}{2 r^{2}} \frac{\partial \tilde{\xi}^{\prime}}{\partial \gamma}+\frac{1}{2 r^{3}} \frac{\partial \tilde{\xi}}{\partial \gamma}
$$

Eq. (22) can be solved for $u(r)$

$$
u=\frac{2}{\tilde{\xi}^{2}} \int_{\infty}^{r} \tilde{\xi}\left(r_{1}\right) F\left(r_{1}\right) d r_{1} .
$$

Eq. (20) can be reformulated as an Euler equation

$$
-\frac{1}{2} \frac{\nabla^{2} \xi^{1 / 2}}{\xi^{1 / 2}}+v_{K S}+u=0
$$

or

$$
-\frac{1}{2} \nabla^{2} \xi^{1 / 2}+\left(v_{K S}+u\right) \xi^{1 / 2}=0
$$

In the original Euler equation there is a constant on the right-hand side of Eq. (25). We can formaly add a constant $\mu$ to both sides of Eq. (25):

$$
-\frac{1}{2} \frac{\nabla^{2} \xi^{1 / 2}}{\xi^{1 / 2}}+v_{K S}+\hat{u}=\mu .
$$

However, $\hat{u}(r)=u(r)+\mu$ is defined only up to a constant.

\section{DISCUSSION}

The derivation in Section III is valid for generalized densities of spherical symmetries. This is the case of filled shells. The findings of the paper also hold for systems with partially 
filled shells, that is, for degenerate states, if we take the spherical part of the generalized density. It was shown by Theophilou $[18,19]$ that the spherical part of the density uniquely defines the external potential. In our case the spherical part of the generalized density should be taken: $\tilde{\xi}(r)=\int d \theta \int d \phi \sin \theta \xi(r, \theta, \phi) /(4 \pi)$. The radial parts $P_{i}$ of the orbitals fulfill Eq. (4) and with $\varrho_{i}=P_{i}^{2}$ the whole derivation is valid.

In the Kohn-Sham scheme of the density functional theory, the non-interacting kinetic energy can be partitioned as a sum of the full Weizsäcker kinetic energy ([20]) and the so-called Pauli energy $T_{p}[21-23]$ :

$$
T_{s}=T_{w}+T_{p}
$$

The functional derivatives are

$$
\frac{\delta T_{w}}{\delta n}=\frac{1}{8}\left|\frac{\nabla n}{n}\right|^{2}-\frac{1}{4} \frac{\nabla^{2} n}{n}=n^{-1 / 2}\left(-\frac{1}{2} \nabla^{2}\right) n^{1 / 2}
$$

and

$$
v_{p}=\frac{\delta T_{p}}{\delta n}
$$

where $v_{p}(\mathbf{r})$ is the Pauli potential. Therefore the Euler equation of the density functional theory

$$
\frac{\delta T_{s}}{\delta n}+v_{K S}=\mu
$$

can be rewritten as

$$
\frac{\delta T_{w}}{\delta n}+v_{p}+v_{K S}=\mu
$$

It can also be written as a Schrödinger-like equation for the square root of the density

$$
\left[-\frac{1}{2} \nabla^{2}+v_{p}+v_{K S}\right] n^{1 / 2}=\mu n^{1 / 2}
$$

The appearence of the Pauli potential [21-23] in the Euler equation (31) is the consequence of the Pauli principle. The density is determined by a single equation (31). All information concerning the shell structure is incorporated into a local potential $v_{p}(r)$.

The generalized radial density is equal to the ground-state radial density if $\alpha_{k}=\lambda_{k}$ or $a_{k}=\lambda_{k}$ and $w_{k}=1$. It corresponds to $\beta=\gamma=0$. For other values of $\beta$ and $\gamma, w_{k}$ is different from 1 . It has been shown in recent papers $[12,13,15]$ that this generalized density 
is similar to an ensemble density. This ensemble interpretation makes it possible to give a solution to the orbital-free problem. Instead of the original radial density depending only on the radial distance, the ensemble radial density (defined as $\sum_{i} \lambda_{i} w_{i} \varrho_{i}(r)$ ) depending also on $\beta$ and $\gamma$ is considered. Making use of Eqs. (22) - (24) for the case of the ensemble radial density, the Euler equation can be solved.

The new Euler equations (26) can be considered the generalizations of the original Euler equation (33). Eq. (26) contains Eq. (33) as a special case: Eq. (26) is equivalent to Eq. (33) if the generalized density is the density itself. The generalized Euler equation (26) contains a generalized Pauli potential $\hat{u}(r)$ resulting from the Pauli principle. The generalized density $\xi(r)$ has also shell structure which is guaranted by the generalized Pauli potential.

The Eqs. (22) - (24) make it possible to obtain an orbital-free solution for the generalized density $\xi(r)$. It can be done as follows:

(i) Starting from an initial guess of the generalized density, the function $F(r)$ is calculated by Eq. (23).

(ii) Using Eq. (24) calculate the generalized potential $u(r)$.

(iii) Solve the generalized Euler equation (26) to obtain the generalized density $\xi(r)$. Note that one needs the Kohn-Sham potential to obtain the solution. The accuracy of the results will depend on the approximate functional used in solving the Euler equation.

(iv) Repeat steps (i)-(iii) until convergence.

Though it seems to be a simple procedure, novel numerical methods are needed because of the $\beta$ and $\gamma$ dependence in $\xi$. For the case where $\xi(r)$ is the density itself, first results on Be atom are already available [24].

As an illustration consider the following generalized densities: the non-interacting energy density and the angular momentum density. The radial non-interacting energy density $\varrho_{\epsilon}(r)$ is defined as

$$
\varrho_{\epsilon}(r)=\sum_{i} w_{i} \lambda_{i} \epsilon_{i} \varrho_{i}(r)
$$

that is, $a_{i}=\lambda_{i} \epsilon_{i}$. It is equal to the partial derivative of the radial density $\varrho(r)$ with respect to $\beta$

$$
\varrho_{\epsilon}=\frac{\partial \varrho}{\partial \beta} .
$$




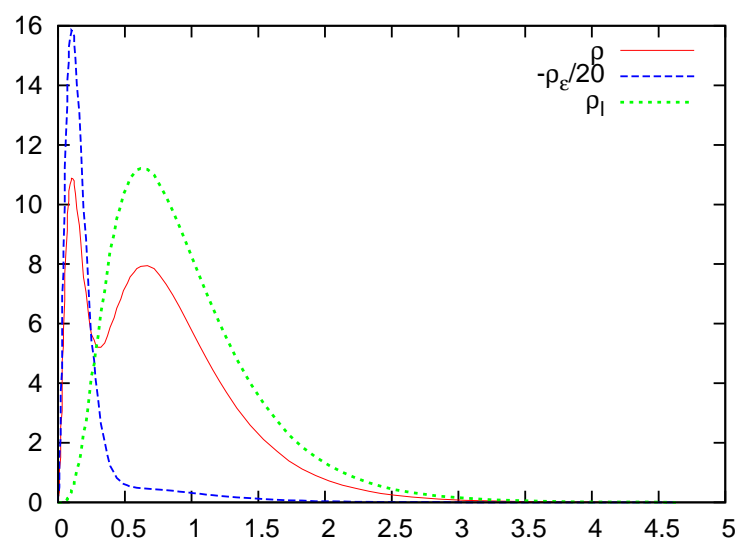

FIG. 1: The radial density $\varrho$, the angular momentum density $\varrho_{l}$ divided by -20 and the angular momentum density $\varrho_{l}$ for $\beta=\gamma=0$ as a function of the radial distance for the Neon atom. (Atomic units).

The radial angular momentum density $\varrho_{l}(r)$ is defined as

$$
\varrho_{l}=\sum_{i} w_{i} \lambda_{i} l_{i}\left(l_{i}+1\right) \varrho_{i}
$$

that is, $a_{i}=\lambda_{i} l_{i}\left(l_{i}+1\right)$. It can be expressed with the partial derivative of the radial density $\varrho(r)$ with respect to $\gamma$

$$
\varrho_{l}=-\frac{\partial \varrho}{\partial \gamma}
$$

To illustrate that not only the density has a shell structure but the non-interacting energy density $\varrho_{\epsilon}(r)$ and the angular momentum density $\varrho_{l}(r)$ also reflect the Pauli principle, $\varrho_{\epsilon}(r)$ and $\varrho_{l}(r)$ are presented in Fig. 1 for $\beta=\gamma=0$ in case of the Ne atom. To a better visibility $-\varrho_{\epsilon}(r) / 20$ is plotted instead of $\varrho_{\epsilon}(r)$. (Atomic units are used throughout in the paper.) For comparison Fig. 1. shows the radial density, too. As it is clear from the definitions, for the Ne atom, $-\varrho_{\epsilon}(r) / 20$ has its maximum, where the radial density takes its first maximum, while $\varrho_{l}(r)$ has its maximum, where the radial density takes its second maximum. Note that the local ionization potential $\tilde{\varepsilon}(\mathbf{r})=\sum_{i} \varepsilon_{i} n_{i}(\mathbf{r}) / n(\mathbf{r})$ introduced by Politzer et al. [25, 26] is closely related to the function $\varrho_{\epsilon}(r)$. The local ionization potential is a measure of chemical reactivity and is linked to the local temperature, and thus to the local kinetic energy [5]. So there is a very intimite relationship between the function $\varrho_{\epsilon}(r)$ and the local kinetic energy.

There are descriptors of the Coulomb systems that do not belong to the family studied in this paper. For example, the Shannon entropy and Fisher information are also capable of 
fully determining every property of a Coulomb system. Finally, in comparison to the Euler equations studied in this paper, the Euler-like equations for specific information that have been derived in a recent paper [27] are summarized. For a system with electron density $n$, the Shannon information can be written as

$$
S=-\int n(\mathbf{r}) \ln n(\mathbf{r}) d \mathbf{r}=\int n(\mathbf{r}) s(\mathbf{r}) d \mathbf{r},
$$

where $s(\mathbf{r})$ is the specific Shannon information ( Shannon information per particle). The Fisher information can also be written as

$$
I=\int \frac{[\nabla n(\mathbf{r})]^{2}}{n(\mathbf{r})} d \mathbf{r}=\int n(\mathbf{r}) i(\mathbf{r}) d \mathbf{r},
$$

where $i(\mathbf{r})$ is the specific Fisher information ( Fisher information per particle). The Euler equation (33) for the density can also been written as

$$
\frac{1}{8}\left|\frac{\nabla n}{n}\right|^{2}-\frac{1}{4} \frac{\nabla^{2} n}{n}+v_{p}+v_{K S}=\mu
$$

The Euler-like equation for the specific Shannon information has the form [27]

$$
\frac{1}{4} \nabla^{2} s-\frac{1}{8}|\nabla s|^{2}+v_{p}+v_{K S}=\mu .
$$

For spherically symmetric systems the Euler-like equation for the specific Fisher information can be written as $[27]$

$$
-\frac{1}{8} i+\frac{1}{2} \frac{i^{1 / 2}}{r}+\frac{1}{4}\left(i^{1 / 2}\right)^{\prime}+v_{p}+v_{K S}=\mu .
$$

The Euler-like equations (41) and (42) have really some resemblance to the original Euler equation(40). The differences are arrising from the definitions of the specific Shannon and Fisher information.

In summary, Coulomb systems have the very special property that not only the groundstate electron density determines all their properties. There are several other quantities that are also capable of fully determining every property of a Coulomb system. Though these descriptors have been known and studied for a while, equations for their calculations have not been presented yet. To fill this gap, Euler equations for a family of descriptors of the spherically symmetric Coulomb systems are derived and discussed. These can be considered the generalizations of the Euler equation of the density functional theory.

[1] P. Hohenberg and W. Kohn, Phys. Rev. 1964, 136, B864. 
[2] P. W. Ayers, Proc. Natl. Acad. Sci 2000, 97, 1959.

[3] P. W. Ayers, Chem. Phys. Lett. 2007, 438, 148.

[4] P. W. Ayers and Á. Nagy, J. Chem. Phys. 2007, 126, 144108.

[5] P. W. Ayers, R. G. Parr and Á. Nagy, Int. J. Quantum Chem. 2002, 90, 309.

[6] Á. Nagy, Chem. Phys. Lett. 2008, 460, 343.

[7] Á. Nagy, Indian J Chem 2014, 53A, 965.

[8] Á. Nagy, Chem. Phys. Lett. 2013, 556 , 355.

[9] E. Chacón, J. E. Alvarellos and P. Tarazona, Phys. Rev. B 1985, 32, 7868.

[10] C. Huang and E. A, Carter, Phys. Rev. B 2010, 81, 045206.

[11] S. B. Trickey, V. V. Karasiev and A. Vela, Phys. Rev. B 2011, 84, 075146.

[12] Á. Nagy, J. Chem. Phys. 2011, 135, 044106.

[13] Á. Nagy, Int. J. Quant. Chem. 2010, 110, 2117.

[14] Á. Nagy, in Recent Advances in Orbital-free Density Functional Theory eds. T. A. Wesolowski and Y. A. Wang, World Scientific, Singapore, 2013.

[15] Á. Nagy, Progress In Electromagnetics Research Symposium Proceedings, PIERS Proceedings, 1913 - 1917, July 6-9, Prague, 2015 http://piers.org/piersproceedings/piers2015PragueProc.php?start $=400$

[16] P. W. Ayers, Theor. Chem. Acc. 2003,110, 267.

[17] W. Kohn and L. J. Sham, Phys. Rev.1965, 140, A1133.

[18] A. K. Theophilou, Int. J. Quant. Chem. 1998, 69, 461.

[19] A. K. Theophilou, AIP Conf. Proc. 2015, 1642, 187.

[20] C. F. Weizsäcker, Z. Phys. 1935, 96341.

[21] N. H. March, Phys. Lett. A 1986, 113, 476.

[22] M. Levy, J. P. Perdew, and V. Sahni, Phys. Rev. A 1984, 30, 2745.

[23] S. B. Liu, J. Chem. Phys. 2007 126, 244103.

[24] H. Levämäki, Á. Nagy, K. Kokko and L. Vitos, Phys. Rev. A in press.

[25] P. Politzer, J. S. Murray, M. E. Grice, T. Brinck and S. Ranganathan, J. Chem. Phys. 1991, 95,6699 .

[26] J. S. Murray, J. M. Seminario, P. Politzer and P. Sjoberg, Int. J. Quantum Chem. 2010, 24, 645 .

[27] Á. Nagy, Int. J. Quant. Chem. 2014, 114, 24812. 


\section{Appendix: Miscellaneous Mathematics}

It is useful to recall some expressions for $\nabla$ and $\nabla^{2}$ in spherical coordinates. Denoting the unit vectors by $\mathbf{e}_{r}, \mathbf{e}_{\theta}$ and $\mathbf{e}_{\phi}$, the $\nabla$ operator acts on an a function $f(\mathbf{r})$ as

$$
\nabla f(\mathbf{r})=\nabla f(r, \theta, \phi)=\frac{\partial f}{\partial r} \mathbf{e}_{r}+\frac{1}{r} \frac{\partial f}{\partial \theta} \mathbf{e}_{\theta}+\frac{1}{r \sin \theta} \frac{\partial f}{\partial \phi} \mathbf{e}_{\phi} .
$$

If the function depends only on $r$

$$
\nabla f(r)=\frac{d f}{d r} \mathbf{e}_{r}
$$

The Laplacian acts on a function $f(\mathbf{r})$ as

$$
\nabla^{2} f(\mathbf{r})=\frac{1}{r^{2}} \frac{\partial}{\partial r}\left(r^{2} \frac{\partial f}{\partial r}\right)+\frac{1}{r^{2} \sin \theta} \frac{\partial}{\partial \theta}\left(\sin \theta \frac{\partial f}{\partial \theta}\right)+\frac{1}{r^{2}(\sin \theta)^{2}} \frac{\partial^{2} f}{\partial \phi^{2}} .
$$

If the function depends only on $r$

$$
\nabla^{2} f(r)=\frac{1}{r^{2}} \frac{d}{d r}\left(r^{2} \frac{d f}{d r}\right)
$$

\section{Acknowledgments}

The work is supported by the Grant OTKA No. K 100590. 